\title{
MULTIPLE DISJOINTNESS FOR WEAKLY MIXING REGULAR MINIMAL FLOWS
}

\author{
DOUGLAS McMAHON
}

\begin{abstract}
We show that pairwise disjointness implies multiple disjointness for metric, weakly mixing regular minimal flows with an abelian phase group. A result on the disjointness of graphic minimal flows is also included.
\end{abstract}

In a recent paper Auslander and Markley studied graphic flows and multiple disjointness for integer actions. Their major result is the following: Given a family of graphic minimal sets $\left(X_{i}, T_{i}\right), T_{i}$ a homeomorphism of $X_{i}$, and nonzero integers $a(i)$, if the flows $\left(X_{i}, T^{a(i)}\right)$ are pairwise disjoint, then the product of all the flows is minimal. A related result is that, given a graphic flow $(X, T)$, then $\left(X, T^{m}\right)$ and $\left(X, T^{n}\right)$ are disjoint whenever $m \neq+/-n$. In this paper we generalize the related result to $Z^{n}$ actions. We intended to generalize the major result to $R^{n}$ actions, but found rather surprisingly that the result held for metric, weakly mixing regular minimal flows with abelian phase group, a substantially stronger result.

The techniques used should be useful for proving generalizations of other results about graphic minimal flows as well as related results in [KN76] and [W75]. I would like to thank Ed Ihrig for useful conversations, Joe Auslander for catching an error in my original manuscript, and the referee for allowing me to include an improved result in the revised copy.

\section{Preliminaries.}

Standing assumptions. We assume that a flow $(X, T)$ has a compact phase space and locally compact abelian phase group. Let $J$ be the set of idempotents in the universal minimal set for $T$.

DEFINITION. A flow $(X, T)$ is a regular minimal flow iff it is a minimal flow such that, for any pair of points $x, x^{\prime}$ with $\left(x, x^{\prime}\right)$ an almost periodic point in $(X, T) \times$ $(X, T)$, there exists an automorphism $h$ of $(X, T)$ with $h(x)=x^{\prime}$.

DEFINITION. A minimal flow $(X, T)$ is weakly mixing minimal iff it has no nontrivial equicontinuous factor. When $X$ is metric this is equivalent to $(X, T) \times$ $(X, T)$ having a point with dense orbit.

DEFINITION. A flow $(X, T)$ is totally minimal iff $(X, S)$ is minimal for all syndetic subgroups $S$ of $T$.

DEFINITION. A flow $(X, T)$ is graphic iff it is a weakly mixing minimal flow, and $X u$ is a single orbit for all idempotents $u$ in $J$ iff it is a minimal flow such that, for any pair of points $x, x^{\prime}$ with $\left(x, x^{\prime}\right)$ an almost periodic point in $(X, T) \times(X, T)$, there exists a $t$ in $T$ with $x t=x^{\prime}$.

Note a graphic flow is a regular minimal flow and is totally minimal by 2.32 of [GH].

First we consider when pairwise disjointness implies multiple disjointness.

Received by the editors October 10, 1984 and, in revised form, September 16, 1985.

1980 Mathematics Subject Classification (1985 Revision). Primary 54H20. 
(1) THEOREM. Let $T$ be abelian and $(X, T),(Y, T)$, and $(Z, T)$ minimal flows with $(X, T)$ a regular minimal flow and $Z$ weakly mixing. If $N \subseteq X \times Y$ is minimal and $(X, T)$ and $(Y, T)$ are disjoint from $(Z, T)$, then $(N, T)$ is disjoint from $(Z, T)$ provided $X, Y, Z$ are metric or $T$ is countable.

ProOF. We first give the proof if the phase spaces are metric. Let $u \in J$ and $(x, y, z) u=(x, y, z)$ where $(x, y)$ is a point in $N$. Let $W$ be the orbit closure of $(x, y, z)$ in $(X, T) \times(Y, T) \times(Z, T)$ (note $W$ is a minimal set). Let $\left\{h_{j}: j \in T\right\}$ be an enumeration of the elements of $T$ and let $S$ be the free group generated by the $j$ 's. Let $S$ act on $Z$ in the natural way, i.e. any element $s$ in $S$ is a finite product of $j$ 's, consider $s$ as a product of the corresponding $h_{j}$ 's as such it is an element of $T$ and has an action on $Z$; also each element of $S$ has only one representation as a product of $j$ 's and so the action is well defined. Note that $(Z, T)$ has an invariant measure since $T$ is abelian and $(Z, S)$ has the same invariant measure. Let $S$ act on $Y$ trivially, i.e. $y s=y$ for all $s$ in $S$ and $y$ in $Y$. Since $(X, T)$ is a regular minimal flow, for each $j$ there exists a homomorphism $B_{j}$ such that $\left(x B_{j}, y, h_{j}\right)$ is in $W$. (Indeed we have $\left(x^{*}, y, z h_{j}\right) \in W$ for some $x^{*}$ by the assumption of minimality of $(Y, T) \times(Z, T)$ and may assume $x^{*}=x^{*} u=x B_{j}$ for some homomorphism $B_{j}$ since $(X, T)$ is a regular minimal flow.) Note if $\left(x^{\prime}, y^{\prime}, z^{\prime}\right) \in W$, so is $\left(x^{\prime} B_{j}, y, z^{\prime} h_{j}\right)$ since $T$ is abelian. Define an action of $S$ on $X$ by considering $s$ in $S$ as a product of the corresponding $B_{j}$ 's; again this gives an action since $S$ is a free group. Note that if $\left(x^{\prime}, y^{\prime}, z^{\prime}\right) \in W$, so is $\left(x^{\prime}, y^{\prime}, z^{\prime}\right) s$.

Now $(X, S)$ is not minimal in general, so let $X^{*}$ be a minimal subset of $(X, S)$ and $x^{\prime} \in X^{*}$. Note that there exists $z^{\prime}$ in $(Z, S)$ such that $\left(x^{\prime}, z^{\prime}\right) S$ is dense in $X^{*} \times Z$ since $(Z, S)$ is weakly mixing minimal and has an invariant measure (see 1.11 of [M78]). In particular, the closure of $\left(x^{\prime}, z^{\prime}\right) S$ contains $\left\{x^{\prime}\right\} \times Z$. Note that $\left(x^{\prime}, y^{\prime}, z^{\prime}\right) \in W$ for some $y^{\prime}$ in $Y$ (and $\left.\left(x^{\prime}, y^{\prime}\right) \in N\right)$ since $(X, T) \times(Z, T)$ is minimal, and so $\left\{x^{\prime}\right\} \times\left\{y^{\prime}\right\} \times Z \subseteq W$. Then since $\left(x^{\prime}, y^{\prime}\right)$ is in the minimal set $N$, we have that for all $\left(x^{*}, y^{*}, z^{*}\right)$ in $N \times Z$ there exists a net with $x^{\prime} t_{n} \rightarrow x^{*}$ and $y^{\prime} t_{n} \rightarrow y^{*}$, so that $\left(x^{\prime}, y^{\prime}, z^{*} t_{n}^{-1}\right) t_{n} \rightarrow\left(x^{*}, y^{*}, z^{*}\right)$. Thus $N \times Z=W$. This proves the theorem if the phase spaces are metric.

Note that in the above the assumption that $X$ was regular was only used to obtain the isomorphisms $B_{j}$, and note that the elements of $T$ commute with the elements of $S$; and so we can consider the action of $S \times T$ on $X, Y, Z$. Now suppose that these flows can be obtained as the inverse limit of flows $\left(X_{n}, S \times T\right)$, $\left(Y_{n}, S \times T\right),\left(Z_{n}, S \times T\right)$. Then $\left(X_{n}, T\right),\left(Y_{n}, T\right),\left(Z_{n}, T\right)$ are metric minimal flows with $\left(X_{n}, T\right)$ and $\left(Y_{n}, T\right)$ disjoint from $\left(Z_{n}, T\right)$. Let $W_{n}$ and $N_{n}$ be the images of $W$ and $N$ and note that the appropriate $B_{j}$ 's exist (they are elements of $S$ ) so that the technique used above works. Thus $\left(N_{n} \times Z_{n}, T\right)$ is minimal and so is the inverse limit $(N \times Z, T)$. By 3.3 of [W67] the flows can be obtained as inverse limits when $S \times T$ is countable, which is the case when $T$ is countable.

(2) COROLLARY. Let $\left(X_{i}, T\right), i$ in some index set $L$, be metric, weakly mixing regular minimal flows. If $\left(X_{i}, T\right)$ and $\left(X_{j}, T\right)$ are disjoint for $i \neq j$, then $\pi\left(X_{i}, T\right)$ is minimal.

Now we consider disjointness for graphic flows.

Let $g$ and $h$ be (commuting) homeomorphisms of $X$ inducing a $Z \times Z$ action $\pi$, then by $x(1,0)=\pi(x,(1,0))$ we mean $x g$ and by $x(0,1)$ we mean $x h$. We can 
combine $h$ and $g$ to give other $Z \times Z$ actions $\pi_{M}$ by letting $x(1,0)=\pi_{M}(x,(1,0))=$ $x g^{m} h^{m^{\prime}}$ and $x(0,1)=x g^{n} h^{n^{\prime}}$ where $M$ is the matrix $\left(\begin{array}{ccc}m & m^{\prime} \\ n & n^{\prime}\end{array}\right)$; this latter action will be denoted by $\left(X, Z \times Z, \pi_{M}\right)$ or $(X, M)$, the original action would be denoted by $(X, I)$ where $I$ is the identity matrix. This latter notation can be extended to any finite number of (commuting) homeomorphisms $g_{1}, g_{2}, \ldots, g_{n}$ giving rise to an $n \times n$ matrix $M$. More generally for any flow $(X, T, \pi)$ and group homomorphism $M: T \rightarrow T$, one can define a new flow $\left(X, T, \pi_{M}\right)$ by $\pi_{M}(x, t)=\pi(x, t M)$. For example, the homomorphism for the $Z \times Z$ action above is $M: Z \times Z \rightarrow Z \times Z$ defined by $\left(z, z^{\prime}\right) M=\left(z m+z^{\prime} n, z m^{\prime}+z^{\prime} n^{\prime}\right)$. Noting some confusion that might arise from notation, we will write $\pi(x, t)=x t$ and $\pi_{M}(x, t)=x t M$, so $x t$ in $(X, T, \pi)$ is the same as in $\left(X, T, \pi_{M}\right)$; while for $u$ in $J, x u=\lim \pi\left(x t_{n}\right)$ in $(X, T, \pi)$ is not the same as $x u=\lim \pi_{M}\left(x t_{n}\right)$ in $\left(X, T, \pi_{M}\right)$. Occasionally, we will denote the first by $x u_{I}$ and the second by $x u_{M}$ when confusion seems likely.

(3) Proposition. Let $M_{1}$ and $M_{2}$ be homomorphisms of $T$ into $T$ and let $I$ be the identity homomorphism. Suppose $\left(X_{j}, I\right)$ are graphic flows, $j=1,2$, $\left(X_{1}, M_{1}\right)$ and $\left(X_{2}, M_{2}\right)$ are minimal, and $T=Z^{n}$ for some integer $n$. Identify the homomorphisms with their integer matrices with respect to the usual basis for $Z^{n}$. Then $\left(X_{i}, M_{i}\right)$ and $\left(X_{2}, M_{2}\right)$ are disjoint whenever $\operatorname{det} M_{1} A-M_{2} \neq 0$ for all choices of integer matrices $A$. In particular, this is the case if $\operatorname{det} M_{2}$ is not congruent to 0 mod the gcd of $M_{1}$.

PrOOF. For convenience we denote the homomorphisms by $M=M_{1}$ and $P=M_{2}$. Let $u \in J$. Let $W$ be the orbit closure of $(x u, x u)=\left(x_{1} u_{M}, x_{2} u_{P}\right)=$ $(x u, x u) u_{M, P}$ under the product flow $\left(X_{1}, M\right) \times\left(X_{2}, P\right)$ (the action is $(x, x) t=$ $(x t M, x t P))$. Note that $W$ is minimal. Let $L=\{t \in T:(x u, x u t) \in W\}$. Then $L$ is a subgroup. To see this note that if $t$ and $t^{\prime}$ are in $L$, then $(x u, x u t)=$ $\lim (x u, x u) t_{n}=\lim \left(x u t_{n} M, x u t_{n} P\right)$, and so

$$
\begin{aligned}
\left(x u, x u t t^{\prime}\right)= & \lim \left(x u t_{n} M,\left(x u t_{n} P\right) t^{\prime}\right)=\lim \left(x u t_{n} M, x u\left(t_{n} P+t^{\prime}\right)\right) \\
= & \lim \left(x u t_{n} M,\left(x u t^{\prime}\right) t_{n} P\right)=\lim \left(x u, x u t^{\prime}\right) t_{n} \in W
\end{aligned}
$$

(where we write $T$ in additive form since it is abelian). To see that $-t$ is also in $L$ if $t$ is in $L$, note that $(x u, x u(-t))$ is an almost periodic point and $(x u, x u)$ is in its orbit closure. If $L$ is syndetic in $T$, then the flows will be disjoint by the total minimality of $\left(X_{2}, I\right)$ since then $\{x u\} \times X_{2} \subseteq W$ and so $\{x u t M\} \times X_{2} \subseteq W$, giving $X_{1} \times X_{2} \subseteq W$.

We wish to give some conditions under which $L$ will be syndetic. We first show there exists a homomorphism $A$ of $T$ into $T$ such that $(x u t, x u t A) \in W$ for all $t$ in $T$. Fix $t$ in $T$, note by the minimality of $(X, I)$ that there is some $y$ for which $(x u t, y) \in W$; then $\left(x u t, y^{*}\right)=(x u t, y) u \in W$ for some $y^{*}=y^{*} u_{P}=y u_{P}$ and so $y^{*}=x u_{P} t^{*}\left(=x_{2} u_{P} t^{*}\right)$ for some $t^{*}$ in $T$ (there may be more than one such $\left.t^{*}\right)$. Now take the usual basis $e_{i}, i=1, \ldots, n$, for $T=Z^{n}$. Then there exist integers $a_{i j}$ such that $\left(x u e_{1}, x u\left(\sum a_{i j} e_{j}\right)\right) \in W$ and the homomorphism $A$ is defined by extending this action $e_{i} \rightarrow \sum a_{i j} e_{j}$ linearly; clearly the matrix representation is $\left\{a_{i j}\right\}$ an integer matrix.

Consider $(x u t M, x u t M A) \in W$. Then

$$
\begin{aligned}
(x u, x u t(M A-P)) & =(x u(t M-t M), x u(t M A-t P)) \\
& =(x u t M, x u t M A)(-t) \in W .
\end{aligned}
$$


So $t(M A-P) \in L$ for all $t$ in $T$. The flows will be disjoint if $T(M A-P)$ is syndetic in $T$, this will be the case if the matrix representation of $M A-P$ has nonzero determinant (we will use the same notation for the matrix as for the homomorphism). Consider $\operatorname{det} M A-P$. We wish to give some condition depending on $M$ and $P$ under which it is not zero for all choices of integer matrices $A$ since we do not have much control over $A$. Let $n=2$ and $d=\operatorname{gcd} M$ denote the greatest common divisor of the entries of $M$. Let $M=d M^{\prime}$ and $H=M^{\prime} A$. Then

$$
\begin{aligned}
\operatorname{det} M A-P & =\operatorname{det} d H-P \\
& =\left(d h_{11}-p_{11}\right)\left(d h_{22}-p_{22}\right)-\left(d h_{21}-p_{21}\right)\left(d h_{12}-p_{12}\right)
\end{aligned}
$$

(using the usual notation for a matrix). Clearly this is congruent to $\operatorname{det} P \bmod d$. Thus the subgroup $L$ is syndetic as long as $\operatorname{det} P$ is not congruent to 0 mod the gcd of $M$. Note the roles of $M$ and $P$ are symmetric.

REMARK. Under a real action the matrix $A$ would not be an integer matrix and could be $M^{-1} P$ if $M$ is invertible, and the above proof would fail.

Note that this theorem is a generalization since if $n=1$, the flows fail to be disjoint only when $M_{2}=+/-M_{i}$; indeed, $M_{1}$ and $M_{2}$ are just integers and equal to their det and gcd, so if they are not disjoint $M_{2}$ is an integral multiple of $M_{1}$, but the result is symmetric and so $M_{2}=+/-M_{1}$.

Let $n=2, T=Z^{2}$. In order that the $Z \times Z$ flows $(X, M)$ and $(X, P)$ be minimal we would in general need that the matrices

$$
M=\left[\begin{array}{cc}
m & m^{\prime} \\
n & n^{\prime}
\end{array}\right], \quad P=\left[\begin{array}{ll}
p & p^{\prime} \\
q & q^{\prime}
\end{array}\right]
$$

be nonsingular. However, the situation where one of the actions is a $Z$ action can be considered by letting $M=\left[\begin{array}{cc}m & 0 \\ n & 0\end{array}\right]$; note how this illustrates the theorem, the action is just that of $T^{\mathrm{gcd} M}$, where $x T=x(1,0)$ and $x S=x(0,1)$. The result is not all inclusive since it does not answer the case $M=\left[\begin{array}{ll}1 & 0 \\ 1 & 0\end{array}\right]$ and $P=\left[\begin{array}{ll}0 & 1 \\ 0 & 1\end{array}\right]$, they are disjoint but $\operatorname{det} M A-P=0$ for all $A$.

Suppose $\operatorname{det} M=1$ and $\operatorname{det} P=1$, then if $A=M^{-1} P, \operatorname{det} M A-P=\operatorname{det} P-$ $P=0$ and if $B=P^{-1} M, \operatorname{det} P B-M=0$, and the result does not apply; they may or may not be disjoint.

The following is an example of a minimal flow $(Y, S)$ that is not graphic and $(Y, S) \times\left(Y, S^{5}\right)$ is not minimal. Let $(X, T)$ be a POD flow, $T$ a homeomorphism. Let $(Y, S)=(X, T) \times\left(X, T^{5}\right), y S=\left(x, x^{\prime}\right) S=\left(x T, x^{\prime} T^{5}\right)$, it is minimal by [AM]. Then $\left(\left(x, x^{\prime}\right) S,\left(x^{\prime}, x^{*}\right) S^{5}\right)=\left(\left(x T, x^{\prime} T^{5}\right),\left(x^{\prime} T^{5}, x^{25}\right)\right)$ and so the orbit closure of $\left(\left(x, x^{\prime}\right),\left(x^{\prime}, x^{*}\right)\right)$ is not $Y \times Y$. Thus $(Y, S) \times\left(Y, S^{5}\right)$ is not minimal.

\section{REFERENCES}

[AM] J. Auslander and N. Markley, Graphic flows and multiple disjointness, Preprint, July 84.

[GH] W. Gottschalk and G. Hedlund, Topological dynamics, Amer. Math. Soc. Colloq. Publ., vol. 86, Amer. Math. Soc., Providence, R.I., 1955.

[KN76] H. Keynes and D. Newton, Real prime flows, Trans. Amer. Math. Soc. 217 (1976), 237-255.

[MW] D. McMahon and T. Wu, Homomorphisms of minimal flows and genralizations of weak mixing, Pacific J. Math. 104 (1983), 401-416. 
[M78] D. McMahon, Relativized weak disjointness and relatively invariant measures, Trans. Amer. Math. Soc. 236 (1978), 225-237.

[W67] T. S. Wu, Homomorphisms in topological dynamics, Bull. Inst. Math. Acad. Sinica, H.-C. Chow 65th anniversary volume, 1967, pp. 53-61.

[W75] , Notes on topological dynamics. II, Distal extension with discrete fibers and prime flows, Bull. Inst. Math. Acad. Sinica 3 (1975), 49-60.

Department of Mathematics, Arizona State University, Tempe, Arizona 85287 